\title{
Changes in pulmonary microvascular permeability accompanying re-expansion oedema: evidence from dual isotope scintigraphy
}

\author{
P D Wilkinson, Jennifer Keegan, S W Davies, J Bailey, R M Rudd
}

\begin{abstract}
The pathophysiological mechanism of pulmonary oedema following rapid reexpansion of a collapsed lung is poorly understood. It has been suggested that the period of collapse or subsequent reinflation produces an increase in pulmonary microvascular permeability. To investigate this, the pulmonary accumulation of the plasma protein transferrin was measured by radiolabelling it in vivo with indium-113m. Plasma protein accumulation was calculated after correcting the accumulation of transferrin for changes in intrathoracic blood distribution by simultaneously monitoring technetium-99m labelled red blood cells. Functional images of plasma protein accumulation were constructed for the lung fields on a pixel by pixel basis. Investigations were performed on 14 subjects after drainage of a pleural effusion $(n=9)$ or evacuation of a pneumothorax $(n=5)$, and on 11 control subjects. Plasma protein accumulation was greater over the regions of lung reexpansion (-0.1-9.6, mean $2.9 \times 10^{-3}$ I min) than over the corresponding region of the contralateral lung $(-1 \cdot 2-0.8$, mean $\left.0.01 \times 10^{-3} / \mathrm{min} ; \mathrm{p}<0.001\right)$. Patients who had undergone re-expansion procedures also had significantly greater plasma protein accumulation than normal controls. Nine of the 14 patients in the reexpansion group had clearly identifiable areas of increased plasma protein accumulation that corresponded to the part of the lung that had been reexpanded; no regional abnormalities were recorded in the control group. These results suggest that the reinflated lung displays abnormal microvascular permeability.
\end{abstract}

Department of Thoracic Medicine, London Chest Hospital, London P D Wilkinson J Keegan

$S$ W Davies

R M Rudd

Department of Nuclear Medicine J Bailey

Address for reprint requests: Dr Paul Wilkinson, London Chest Hospital, London E2 9JX.

Accepted 21 February 1990 volume of re-expansion, duration of the preceding collapse, and to the degree to which intrapleural pressure is reduced by the application of suction. ${ }^{23}$

Re-expansion pulmonary oedema is recogas an uncommon complication attended by considerable respiratory and circulatory embarrassment. ${ }^{145}$ The likelihood
The aetiology of the oedema has not been determined. One of the cardinal questions is whether there are localised changes in pulmonary microvascular permeability in reexpanded lungs. To address this question, we have attempted to measure the microvascular permeability in re-expanded and normal lungs by means of dual isotope scintigraphy. We used a modification of a non-invasive method described by Gorin and $\mathrm{Staub}^{67}$ and adapted by Basran and colleagues to assess microvascular permeability in the adult respiratory distress syndrome. ${ }^{89}$ In essence, the pulmonary accumulation of the plasma protein transferrin is monitored by radiolabelling it in vivo with indium-113m. In conditions of normal microvascular integrity transferrin is effectively contained within the vascular compartment, or at least escapes from it at a very slow rate; when the pulmonary capillaries are abnormally permeable transferrin accumulates by escaping into the extravascular, extracellular space. Measurements were made in normal subjects and in patients who had had one lung re-expanded after either a pleural effusion or a pneumothorax. The aim was to determine whether abnormalities of microvascular permeability could be detected in re-expanded lungs even when clinical evidence of oedema was not present.

\section{Methods}

The study was approved by the hospital ethical committee and all patients gave their informed, written consent. Studies were performed in $\mathbf{1 4}$ patients after drainage of a pleural effusion (nine patients) or evacuation of a pneumothorax (five patients). Three of the patients had chronic airflow limitation (forced expiratory ratio $<0 \cdot 75$, forced expiratory volume in one second $<75 \%$ predicted, and, in two cases, radiological evidence of emphysema). One patient had systemic sclerosis.

The control group comprised 11 subjects with mild coronary artery disease whose left ventricular function was not appreciably impaired as judged by ventriculography and normal end diastolic pressures of $<10 \mathrm{~mm} \mathrm{Hg}$. All had normal lung function and chest radiographs.

Transferrin was radiolabelled in vivo with $100 \mathrm{MBq}$ of indium-113m chloride from a sterile generator (Amersham International). The in vivo labelling of autologous red cells was accomplished by the intravenous injection of $0.02 \mathrm{mg} / \mathrm{kg}$ body weight of stannous fluoride 
and $0.034 \mathrm{mg} / \mathrm{kg}$ of sodium medronate, followed 20 minutes later by $100 \mathrm{MBq}$ of technetium-99m sodium pertechnetate. The labelling efficiency of this technique is known to be around $70 \%$ (Amersham International, private communication), but was not measured in this study. The fraction of the blood pool technetium bound to red cells is appreciably higher than this figure, however, because of the rapid uptake of free pertechnetate by organs such as the thyroid: a spot measurement, made 15 minutes after injection in one patient, showed $92 \%$ of the technetium to be bound to red cells. The total radiation dose from the two isotopes is estimated to have been $2 \cdot 13 \mathrm{mSv}$, which falls well inside the safety limits stipulated by the World Health Organisation for research projects in which members of the public take part (category II of the WHO classification).

Recordings were made from patients in the supine position with a large field gamma camera positioned underneath the patient facing the posterior aspect of the chest and the portable probe mounted on a secure gantry over the anterior chest wall. The probe was used to record data alternately from the cardiac region and from a fixed position over the lung, the right upper zone (RUZ) for the control group and the area of re-expansion for the other subjects. Appropriate corrections were made for down scatter.

Serial dual energy gamma camera images were recorded over five minute periods during a total scanning time of about 90 minutes. Probe data for both isotopic species were recorded simultaneously. From these data lung

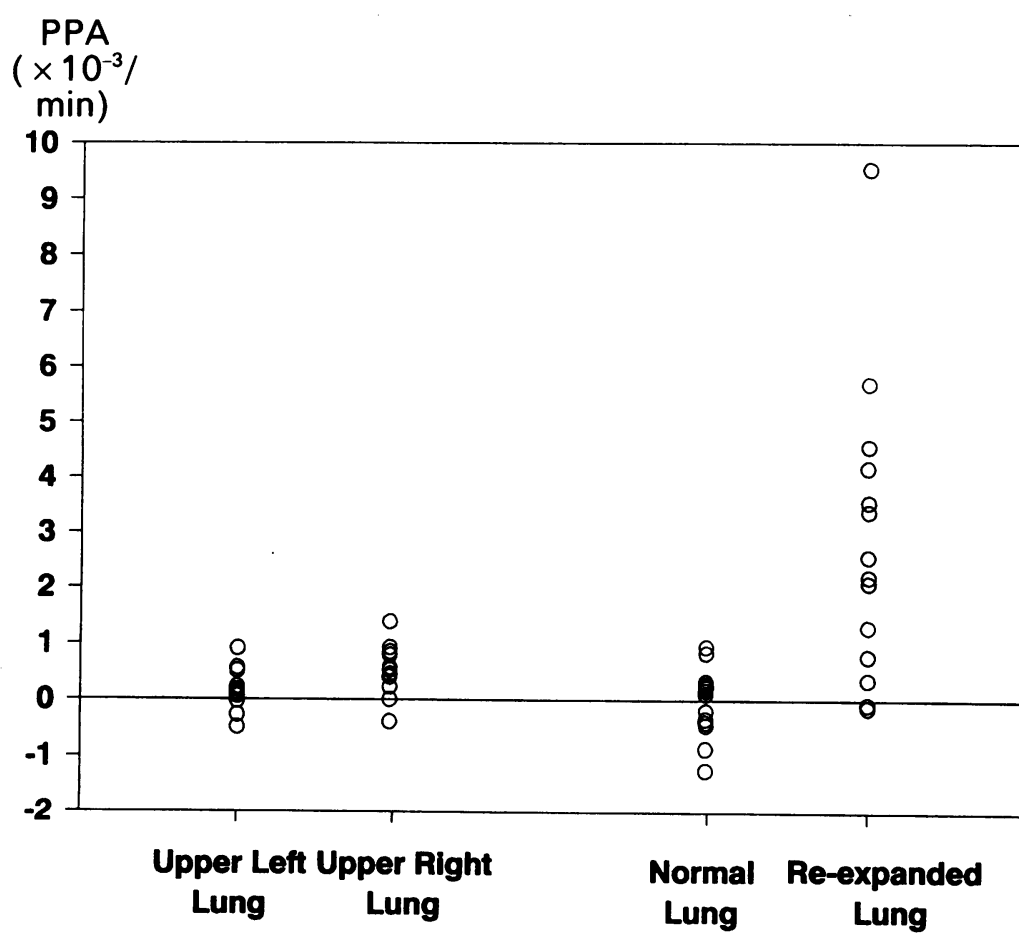

Figure 1 Plasma protein accumulation (PPA) indices calculated from regions of interest, 4 pixels by 4 pixels, selected from whole lung functional images obtained by dual
isotope scintigraphy. For the 11 control subjects plasma protein accumulation indices from the upper zones of right and left lungs are shown; in the 14 patients with reexpanded lungs PPA indices for the re-expanded lung are compared with the corresponding regions of the contralateral lung. transferrin indices (LTI) were calculated according to the formula

$$
\frac{{ }^{113 m} \text { In lung count } /{ }^{113 m} \text { In heart count }}{{ }^{99 m} \mathrm{Tc} \text { lung count } /{ }^{99 \mathrm{~m}} \mathrm{Tc} \text { heart count }}
$$

This index corrects for both radioactive decay and variation in the intrathoracic blood pool during data acquisition. The plasma protein accumulation index, defined as the rate of change of the lung transferrin index with respect to time, is interpreted as a measure of pulmonary vascular permeability. Plasma protein accumulation indices were calculated on a pixel by pixel basis from the data acquired from the whole lung fields by gamma camera; the results enabled a functional image of the lungs to be constructed to display details of regional variation in microvascular permeability.

Indices from the probe readings calculated in a similar way served as a check on the accuracy of the gamma camera data.

\section{ANALYSIS}

Regions of interest, 4 pixels by 4 pixels, were selected from the whole lung functional images, and the data from these selected areas used to calculate plasma protein accumulation indices. The index for each reinflated lung was compared with the index for the corresponding region of the contralateral lung in the same patient (that is, corresponding $4 \times 4$ pixel regions of the right and left lungs were compared). Where interference from the cardiac signal prevented exact matching of areas to be paired the region of closest approximation was chosen. Values were compared by means of Wilcoxon's rank sum test. The plasma protein accumulation indices in patients and control subjects were compared by using the Mann-Whitney $U$ test on the combined values from the right and left lung.

\section{Results}

The patients studied after lung re-expansion comprised 12 men and 2 women, aged 29-93 (mean 65) years. The control group of five men and six women were aged $35-65$ years, with a mean of 56 years.

Plasma protein accumulation values over the re-expanded lung $(-0 \cdot 1-9 \cdot 6$, mean $2.9 \times 10^{-3} / \mathrm{min}$ ) were significantly higher than the values obtained from the opposite lung $\left(\times 1.2-0.8\right.$, mean $0.01 \times 10^{-3} / \mathrm{min}$; p $<0.001$ )-see figure 1 and table. Scrutiny of the data for each isotope showed that the difference was due to relative increases in indium- $113 \mathrm{~m}$ over the re-expanded lung rather than to a fall in the technetium-99m label.

Examination of the results from the control group, however, showed a potential source of bias in that there was a small, but statistically significant, difference in plasma protein accumulation in the right and left lungs, the values from the right side tending to be slightly 
Comparison of the plasma protein accumulation indices (means and ranges) for the reexpanded lungs with values for the contralateral lungs of the same patients and for the left and right lungs of the control subjects: original indices and values corrected for asymmetry ${ }^{\star}$

\begin{tabular}{llc}
\hline & \multicolumn{2}{l}{ Plasma protein accumulation $\left(\times 10^{-3} / \mathrm{min}\right)$} \\
\cline { 2 - 3 } & Uncorrected & After correction \\
\hline Control (mean of left and right lungs) & $0.35(-0.5$ to 1.4$)$ & $0.2(0.5$ to 1.1$)$ \\
$\begin{array}{l}\text { Re-expanded lungs } \\
\begin{array}{l}\text { Contralateral lungs of patients } \\
\quad \text { undergoing re-expansion }\end{array}\end{array}$ & $2.9(-0.1$ to 9.6$)$ & $2.7(-0.1$ to 9.3$)$ \\
\hline
\end{tabular}

*Values for right lungs only reduced by $0.3 \times 10^{-3} / \mathrm{min}$.

larger. As the re-expanded lungs were predominantly right sided the data were reanalysed to allow for this, the mean difference in plasma protein accumulation between the right and the left lung in the control subjects being used as a correction factor. Thus all plasma protein accumulation indices from right lungs were reduced by the mean difference of $0.3 \times 10^{-3} / \mathrm{min}$. The difference between the re-expanded and the normal lung was sustained ( $p<0.001)$ after this correction (table).

The plasma protein accumulation index in the patients (mean of left and right lungs) was higher than the values seen in the control subjects $(p<0.001)$. The difference remained significant at the $0.1 \%$ level when correction for lung asymmetry was introduced.

Scrutiny of the images from each lung, scaled to the same maximum value, showed clear areas of abnormality, corresponding to the regions of lung that had been re-expanded (fig 2). Nine of the 14 patients from the re-expansion group had easily identifiable abnormalities on their functional images and the areas of high plasma protein accumulation always coincided with the part of the lung that had been reinflated. No abnormalities were seen in any image from the control group $\left(\chi^{2}=11.05, \mathrm{p}<0.001\right)$. Only one patient had chest radiogràphic evidence of oedema in association with the abnormality on the functional image.

The duration of lung collapse before expansion could not be estimated adequately in

Figure 2 Functional images of plasma protein accumulation (PPA) values calculated on a pixel by pixel basis. All images are scaled to the same maximum, and the small encircled area represents the cardiac region. The "negative" image (a) of uniformly low plasma protein accumulation is from a patient in the control group. The white area in image (b) reflects high

$P P A$ values corresponding to a region of reexpansion. several patients in this study (particularly those with a pleural effusion). A prolonged period of collapse did not always lead to a positive scan.

\section{Discussion}

The generation of any form of pulmonary oedema depends on the interplay of three principal factors: the difference in hydrostatic pressure between the lumen of the capillaries and the immediate surround, the permeability of the capillary membrane, and the colloid osmotic pressure. ${ }^{10}$ Attempts to formulate a mechanism for re-expansion oedema have focused on the first two of these factors. One possibility is that a net egress of fluid from the microvasculature is brought about by a reduced interstitial pressure. This might be transmitted by application of suction to the pleural cavity, or it might arise through an increase in the elastic recoil of the lung as a result of diminished surfactant after lung collapse. ${ }^{112}$ Another proposed mechanism invokes changes in the compartmentalisation of vascular fluids through neural factors akin to the massive sympathetic discharge that has been implicated in neurogenic oedema. ${ }^{13}$ Yamazaki and colleagues, however, have presented evidence suggesting that the pulmonary blood pool is if anything reduced in a re-expanded lung, and this does not favour the idea of there being pulmonary venous congestion. ${ }^{14}$

A local increase in microvascular permeability represents a further possibility, for which there is some evidence in animal models. ${ }^{15}$ As the lining of pulmonary capillaries comprises a single layer of endothelial cells joined at their edges by tight junctions, the integrity of this layer could well be disrupted by anoxia or distortion during lung collapse. This idea is supported by our finding of higher plasma protein accumulation indices in re-expanded lungs than in the opposite lung of the same patient or in normal controls. Furthermore, the functional images showed these changes to be localised to the region of the lung that had been reinflated.
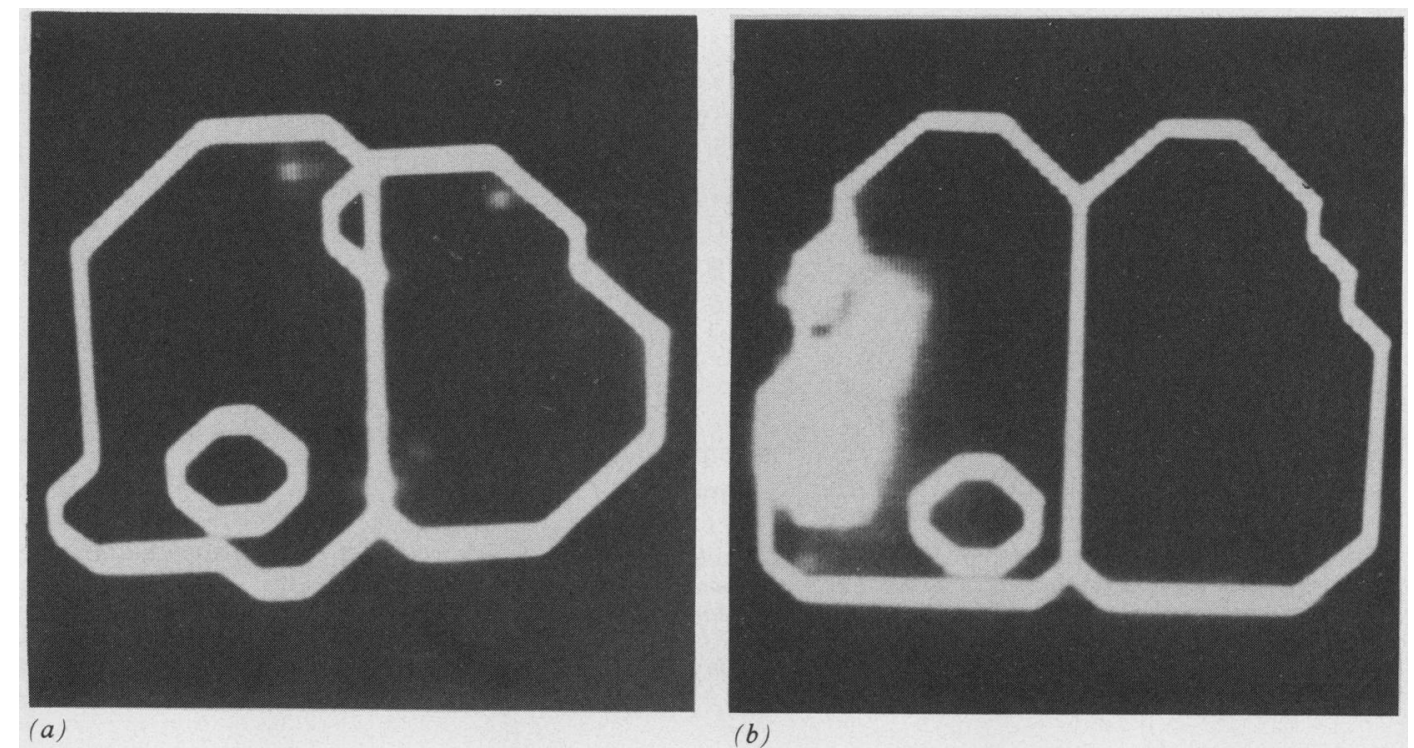
Before we draw firm conclusions, however, we must consider whether the scanning technique was truly registering changes in pulmonary microvascular permeability or merely detecting pleural inflammation caused, for example, by the introduction of an intrapleural drain. It is difficult to be categorical on this point, but the possibility seems unlikely for several reasons. Firstly, four of the five patients whose scans showed no abnormality had had an intrapleural drain inserted. Secondly, the changes observed on functional images were spread more diffusely than would be likely for the presumably localised inflammation caused by a chest drain. Lastly, the idea that an underlying inflammatory condition might have been responsible for the changes cannot account for the very high plasma protein accumulation values found in some patients with an uncomplicated pneumothorax.

The reason for "negative" scans in five patients from the re-expansion group is a matter for conjecture, but it may be relevant that all five patients were either scanned more than 24 hours after the drainage procedure or had undergone a previous partial drainage procedure within the preceding few days. There may have been time therefore for the permeability changes to subside, or the effect of re-expansion may have been reduced by the prior partial reinflation. The nine patients whose functional images showed obvious regional abnormalities all had the scanning done on the day of the primary drainage procedure (and in all but one case within six hours). The numbers are too small, however, to allow firm conclusions on this point.

Our findings suggest that regions of lungs reinflated after a period of collapse often have abnormal microvascular permeability despite the rarity of clinical manifestations of oedema. The state of abnormal microvascular permeability may be short lived, probably lasting for less than 24 hours after re- expansion. These experiments do not provide any indication of whether other factors may also be important in the development of reexpansion oedema, or indeed whether the permeability effects are the "prime mover" or merely secondary to some more fundamental mechanism. Primary damage to the endothelial cells as a direct result of the collapse or through the sudden stretching during rapid reexpansion would nevertheless be a sufficient explanation of the observed pathophysiological effects.

1 Henderson AF, Banham SW, Moran F. Re-expansion pulmonary oedema: a potentially serious complication of delayed diagnosis of pneumothorax. $\mathrm{Br}$ Med $J$ 1985;291:593-4.

2 Mahajan VK, Simon M, Huber L. Re-expansion pulmonary edema. Chest 1979;75:192-4.

3 Waqaruddin $M$, Bernstein A. Re-expansion pulmonary oedema. Thorax 1975;30:54-60.

4 Pavlin JD, Raghu G, Rogers TR, Cheney FW. Reexpansion hypotension. A complication of rapid evacuation of prolonged pneumothorax. Chest 1986;89:70-4

5 Sautter RD, Dreher WH. MacIndoe JH, Meyers WO, Magnin GE. Fatal pulmonary edema and pneumonitis after re-expansion of chronic pneumothorax. Chest 1971;60:399-401.

6 Gorin AB, Weidner WJ, Demling RH, Staub NC. Noninvasive measurement of pulmonary transvascular protein flux in sheep. J Appl Physiol 1978;45:225-33.

7 Gorin AB, Kohler J, DeNardo G. Noninvasive measurement of pulmonary transvascular protein flux in normal man. J Clin Invest 1980;66:869-77.

8 Basran GS, Byrne AJ, Hardy JG. A non-invasive technique for monitoring lung vascular permeability in man. Nucl Med Commun 1985;3:3-10.

9 Basran GS, Byrne AJ, Hardy JG, Richardson MA. The effect of methyl prednisolone on the pulmonary accumulation of transferrin in the adult respiratory distress syndrome. Eur J Respir Dis 1986;68:336-41.

10 Snashall PD. Pulmonary oedema. Br J Dis Chest 1980;74: $2-22$.

11 Childress ME, Moy G, Mottram M. Pulmonary edema resulting from treatment of spontaneous pneumothorax. Am Rev Respir Dis 1971;104:119-21.

12 Trapnell DH, Thurston JGB. Unilateral pulmonary oedema after pleural aspiration. Lancet 1970;i:1367-9.

13 Wauchob TD, Brooks RJ, Harrison KM. Neurogenic oedema. Anaesthesia 1984;39:529-34.

14 Yamazaki S, Ogawa J, Shohzu A, Suzuki Y. Pulmonary blood flow to rapidly re-expanded lungs in spontaneous pneumothorax. Chest 1982;81:118-20.

15 Pavlin DJ, Nessly ML, Cheney W. Increased pulmonary micro-vascular permeability as a cause of re-expansion oedema in rats. Am Rev Respir Dis 1981;124:422-7. 